\title{
Diseño de producción para la agroindustria hortofrutícola de pulpas congeladas, deshidratados, y/o moliendas en la región Caribe
}

\author{
$\triangle$ Edwin Causado Rodríguez ${ }^{1}$ \\ OSCAR JosÉ OSPINO AYALA ${ }^{1}$ \\ JELIBETh PAOLA RACEDO GutiÉRREZ ${ }^{2}$
}

\section{Resumen}

El sector agroindustrial hortofrutícola en Colombia se ha considerado una vía para el progreso, el desarrollo, y la paz; sin embargo, la mayoría de establecimientos procesadores de este sector productivo carecen de un buen esquema de instalaciones, por esto, en el presente artículo se realiza la definición de lineamientos que permita iniciar el proceso para la definición de este tipo de infraestructura productiva, dado que son muy pocos los estudios planteados al respecto, sobre todo en la región Caribe; para lo cual, se propone una metodología para el diseño de instalaciones de plantas agroindustriales. El resultado de esta aplicación evidenció que la planificación del esquema productivo antes de cualquier montaje de plantas, garantiza el cumplimiento de las normas gubernamentales, disminuye las perdidas por mala distribución y asignación de espacios, e incide en el mejoramiento del proceso de manufacturera de manera sustancial. Apreciándose así, que el uso de las herramientas propias de la ingeniería industrial permite el aumento competitivo en las plantas procesadoras de alimentos.

Palabras claves: Matriz Origen-Destino; Diagrama de relaciones; Diseño de instalaciones; Agroindustria hortofrutícola; Proceso de deshidratado; Proceso de despulpado.

1 Universidad del Magdalena,. Santa Marta D.T.C.H, Colombia.

2 Universidad de Los Andes. Bogotá D.C., Colombia.

Autor de correspondencia: Causado Rodríguez (Edwin): Carrera 32 N$^{\circ}$ 22-08 C.P.470004 Santa Marta D.T.C.H.Colombia. Teléfono: 5 -4217940, extensión 1032. Correo electrónico: edwincausado@gmail.com
Historia del artículo:

Artículo recibido: 25-I-2019 / Aprobado: 30-V-2019

Disponible online: 3 de julio de 2019

Discusión abierta hasta marzo de 2021 


\title{
Production Design For The Horticultural Agro-Industry of Frozen, Dehydrated Pulp, And / Or Grinding In The Caribbean Region
}

\begin{abstract}
The agroindustrial horticultural sector in Colombia has been considered a way for progress, development, and peace; However, the majority of processing establishments in this productive sector lack a good scheme of facilities, therefore, in this article, the definition of guidelines is made to start the process for the definition of this type of productive infrastructure, given that There are very few studies on this subject, especially in the Caribbean region; for which, a methodology for the design of agroindustrial plant installations is proposed. The result of this application showed that the planning of the productive scheme before any assembly of plants, guarantees compliance with government regulations, decreases losses due to maldistribution and allocation of spaces, and affects the improvement of the manufacturing process in a substantial manner. Appreciating itself, that the use of the tools of industrial engineering allows the competitive increase in food processing plants.
\end{abstract}

Keywords: Origin-Destination Matrix; Relationship diagram; Design of facilities; Fruit and vegetable agro-industry: Dehydrated process; Pulping process.

\section{Projeto de produção para a agroindústria hortícola de polpa desidratada, congelada e / ou moagem na região do Caribe}

\section{Resumo}

O setor de horticultura agroindustrial na Colômbia tem sido considerado um caminho para o progresso, desenvolvimento e paz; No entanto, a maioria dos estabelecimentos de processamento nesse setor produtivo carece de um bom esquema de instalações, pois, neste artigo, a definição de diretrizes é feita para iniciar o processo de definição desse tipo de infra-estrutura produtiva, dado que Existem poucos estudos sobre esse assunto, especialmente na região do Caribe; para o qual é proposta uma metodologia para o projeto de instalações de plantas agroindustriais. 0 resultado desta aplicação mostrou que o planejamento do esquema produtivo antes de qualquer montagem de usinas, garante a conformidade com as regulamentações governamentais, diminui as perdas devido à má distribuição e alocação de espaços, e afeta a melhoria do processo de fabricação de maneira substancial. . Apreciando-se, que o uso das ferramentas de engenharia industrial permite o aumento competitivo em plantas de processamento de alimentos.

Palavras-chave: Matriz Origem-Destino; Diagrama de relacionamento; Design de instalações; Agroindústria de frutas e vegetais; Processo de desidratação; Processo de polpação. 


\section{Introducción}

El sector agroindustrial hortofrutícola que corresponde a la producción de "bienes de origen agropecuario, como frutas frescas, vegetales y granos, hasta la transformación industrial de bienes como jugos, enlatados, mermeladas, compotas, pulpas, y salsas", se ha convertido para Colombia en un componente productivo clave, por su potencialidad en la obtención de nuevas oportunidades de negocio para los empresarios, cooperativas y ciudadanía organizada (DNP, 2004; PROCOLOMBIA, 2016; Gobernación del Magdalena, 2016). Además aporta, en la disminución de la poli-victimización y en la consecución de la paz de Colombia, mediante la generación de recursos; y la construcción de alianzas que permitan materializar el progreso social, la economía competitiva, y el buen gobierno (DNP, 2015; Campos-Arias, et al., 2016). Lo anterior se ve reflejado, en su pujante de contribución en la generación de empleos, pues, para el 2016 el sector generó 527 mil empleos directos, que superan en $11 \mathrm{mil} \mathrm{em-}$ pleos la cantidad registrada en el 2015, teniendo así, un 21\% de participación en el total de empleos agrícolas en el 2016 (ASOHOFRUCOL, 2016).

Pese a esto, la productividad nacional "ha estado enfrentando un proceso de desindustrialización relativamente acelerado (frente al del mundo desarrollado) desde mediados de la década de los años setenta", sin muchos avances de tecnología, modernización y transformación (Clavijo, Vera y Fandiño, 2012; Palacio, 2014). Lo cual ha repercutido en que el sector agroindustrial hortofrutícola tenga un avance lento, que se evidencia en el incipiente aumento y las condiciones ingenieriles de las plantas procesadoras hortofrutícolas con el transcurrir de los años; su número pasa de 127 organizaciones a 195 entre los años 1993-2001; así mismo, en el año 2006, el número de agroindustriales hortícolas aumentó a 233 establecimientos y en el año 2017 la cantidad de organizaciones superó ligeramente las cifras anteriormente mencionadas (DNP, 2004; MADR, et al., 2006; INVIMA, 2017). Además, con res- pecto a la tecnificación, este incremento se ha realizado de una forma un tanto desorganizada, pues, las plantas de alimentos en su mayoría carecen de la aplicación de herramientas y técnicas de la ingeniería industrial, en términos de diseños productivos; no teniendo una secuencia productiva lógica ingenieril. Es por ello, que PROCOLOMBIA ${ }^{2}$ (2016), plantea la necesidad de incrementar la promoción de este sector a niveles internacionales; como también apoyar decididamente los 30 establecimientos agroindustriales hortofrutícolas que poseen un respaldo ingenieril apropiado a nivel nacional, para de esta manera visibilizar las bondades financieras de estos, como un buen atractivo para atracción de posibles inversiones extranjeras aliadas.

En este escenario, existen investigaciones que buscan fortalecer este aspecto descuidado del diseño de instalaciones del sector en mención. En el entorno internacional, están los autores Kusiak y Heragu (1987); Meyers y Stephens (2006; Watanapa y Wiyaratn, (2011); Lin, et al., (2013); y a nivel nacional los estudios de Causado-Rodríguez, Díaz y Sánchez (2018) quienes presentan la aplicación teórica-práctica de las herramientas estadísticas y gráficas necesarias para efectuar un layout (diseño) adecuado según la necesidad del establecimiento, con la firme intención de disminuir los posibles costes de la empresa por concepto de mal diseño, accidentes, y/o incumplimientos de normativas vigentes.

También, se tienen otros estudios como los realizados por Nariño, et al. (2010), quienes relacionan (para las plantas de alimentos hortalizas y frutas), la distribución en planta con la localización, presentado la importancia de la descripción de la secuencia del proceso y la cercanía de las localidades, para el diseño del establecimiento; así mismo, Ojaghi, et al., (2015), muestran que el buen rediseño requerido de una planta puede lograr un índice de eficiencia del $94,78 \%$ en relación al antiguo del 90,46\%, demostrando así la importancia de la

1. Es la entidad encargada de promover el Turismo, la Inversión Extranjera en Colombia, las Exportaciones no minero energéticas y la imagen del país. 
planificación del diseño en el montaje de la planta. En este mismo ámbito, el documento de Martínez, et al. (2015), los cuales expresan que un buen estudio técnico que incluye el diseño y la logística de la planta, puede ser la respuesta a la satisfacción del cliente evitando desperdicios.

En el contexto local, existen referencias que abordan estudios de diseños productivos en plantas procesadoras de alimentos de hortalizas y frutas ubicadas en zonas con características privilegiadas para la hortofrutícultura, por su fisiografía e hidrografía, como el departamento del Magdalena (INVEMAR, 2004; Meisel-Roca y Pérez-Valbuena, 2006). Una de ellas tuvo como finalidad el mejoramiento productivo de la empresa Bananitas S.A.S. de Santa Marta, logrando obtener mejoras vitales en el diseño actual de la empresa y aumento en la capacidad productiva del 14,7\%, demostrando así la utilidad de la gerencia de operaciones (Corena y $\mathrm{Cu}$ jia, 2012). Así mismo, la investigación de Causado y Reatiga (2013), que presentan el mejoramiento productivo con base a la adecuación de las maquinarias en áreas productivas de la empresa.

En lo anterior, se evidencia una muestra de las aplicaciones del diseño de producción (que puede definirse, como la forma de organizar las instalaciones de la fábrica en el piso de producción para mantener el flujo de manufactura sin problemas), y la necesidad de su uso en el sector agroindustrial hortofrutícola (Sugiyono, 2006). A su vez, la generación de conocimiento y el sector empresarial hortofrutícola, demandan este tipo de estudios que permitan el mejoramiento industrial y la disminución de los costos. Por ello, en la presente investigación, se plantea un diseño de producción desde la región Caribe Colombiana para la transformación productiva con agregación de valor a frutas y hortalizas, en los productos de pulpa de mango congelada (mango de hilaza), berenjena, mango deshidratados (mango de azúcar), y ají molido, por ser este tipo de procesos muy demandados en el mercado internacional (DANE, 2017; MADR, 2016), y además, porque permiten abarcar un mayor número de transfor- maciones productivas, empleadas en la tecnología de alimentos para la agroindustria hortofrutícola. Ofreciendo así, una ayuda táctica a las empresas del sector en mención, y a la comunidad científica una aplicación del diseño de producción que pueda ser base para futuros estudios.

\section{Materiales y Métodos}

Esta investigación cuenta con un enfoque cuantitativo y su alcance es descriptivo. Como fuentes secundarias, se hizo una búsqueda exhaustiva en las bases de datos disponibles por gremios, asociaciones y órganos gubernamentales de Colombia, de las cuales se resaltan: La Asociación Nacional De Empresarios de Colombia (ANDI), el Instituto Colombiano Agropecuario (ICA), el Ministerio de Agricultura y Desarrollo Rural (MADR), la Asociación Nacional de Instituciones Financieras (ANIF), las cámaras de comercio de cada departamento de la región Caribe, PROCOLOMBIA, el Instituto Nacional de Vigilancia de Medicamentos y Alimentos (INVIMA), la Corporación Colombiana de Investigaciones Agropecuaria (CORPOICA), la Dirección de Impuestos y Aduanas Nacionales (DIAN), la Superintendencia de Industria y Comercio, la Superintendencia de Sociedades, el Departamento Administrativo Nacional de Estadística (DANE), el Departamento Nacional de Planeación (DNP), la Sociedad de Agricultores de Colombia (SAC), la Asociación Hortofrutícola de Colombia (ASOHOFRUCOL) y la Federación Colombiana de Productores de Mango (Fedemango). Hallándose que no existen estudios recientes y avalados, que cuantifiquen y midan la fortaleza agroindustrial hortofrutícola en diseño productivo de las plantas procesadoras de la región Caribe. Solo la base de datos de INVIMA (2017), posee datos censales que muestran la cantidad de plantas procesadoras de alimentos que existen en cada departamento del país, aunque no describe la fortaleza manufacturera de estas.

Así mismo se determinó el tamaño de la población a considerar, con base en los conceptos de la 
Resolución Nº 1471 (1984) del Ministerio de Salud, que define el tipo de productos que generan las plantas agroindustriales de frutas y hortalizas, se depuro el censo ofrecido por el INVIMA, lo que arrojó un universo de 50 plantas procesadoras hortofrutícolas en la Región caribe, donde, el 52 \% de los establecimientos se encuentran en Atlántico; $20 \%$ en Bolívar; $10 \%$ en el Magdalena; 8\% en Cesar; $6 \%$ en Córdoba; $2 \%$ en Sucre, y, 2\% en San Andrés Islas y providencia.

Para la aplicación del diseño de instalaciones productivas al sector en estudio, se empleó una metodología de fácil aplicación, la cual consta de las siguientes fases:

\subsection{Fase 1: Aplicación de instrumentos y técnicas para la recolección de información}

Al no existir una valoración actual de la fortaleza agroindustrial de los establecimientos procesadores hortofrutícolas, se realiza la recolección de información primaria, con la finalidad de obtener los requerimientos funcionales y demás, que debe atender el diseño de manufactura. El cual para su alcance cuenta con las actividades descritas a continuación:

\subsubsection{Diseño del cuestionario y medio de aplicación}

Este tiene una estructura que permite delimitar el diseño de instalaciones a unas directrices que responden a los requerimientos del sector en consideración. La herramienta en su contenido cuenta con dos secciones, la primera: obtiene los datos de identificación de la empresa, y en la segunda: mide la fortaleza agroindustrial de la empresa, en vista al diseño de producción.

\subsubsection{Análisis de la información}

Se emplean las técnicas estadísticas de datos agrupados, para obtener una lista de los requerimientos funcionales y demás, a los cuales debe responder el diseño productivo.

\subsection{Fase 2: Determinación de los} patrones de flujo de materiales

Este es un estudio para conocer el recorrido más corto de la materia prima, junto a sus materiales en el diseño de instalaciones. De acuerdo con Niebel, Freivalds y Osuna (2004), los diagramas y gráficos a emplearse se deben definir preliminarmente a criterio del ingeniero en diseño, seleccionándose aquellos que aporten información valiosa al proyecto, para esta fase se tienen las siguientes actividades:

\subsubsection{Gráfico Origen-Destino o} diagrama de-hacía entre máquina

Es el diagnóstico de los problemas relacionados con el arreglo de los departamentos y las áreas de servicios, al igual que con la localización de equipo en un sector dado de la planta (Stephens y Meyers, 2013). Primero, se realiza la codificación de maquinaria empleada en los procesos de interés; segundo, se plasma la ruta de viaje que tienen los diferentes productos a través de las diferentes máquinas asociados al proceso, y se expresa el número de viajes (frecuencia absoluta) que sigue cada producto por cada máquina; y tercero, se realiza el diagrama que presenta una matriz, que tiene como variables de entrada las máquinas necesarias para obtener los productos terminados.

\subsubsection{Díagrama de relaciones de actividades}

Este diagrama muestra la relación que existe entre cada área de los distintos procesos, con la finalidad de conocer que tan importante es la cercanía de un departamento, respecto a otro; ofreciendo así una gran ayuda en los diseños de planos (Stephens y Meyers, 2013). Los autores referentes, tales como: Tak y Yadav, (2012); Ojaghi, et al., (2015); Heragu, (2016); y Maina, Muchiri y Keraita, (2018), recomiendan las siguientes tareas para su construcción:

- Valores de cercanía: estos se encuentran estandarizados en la literatura, así: $A$ (Absolutamente necesario), $E$ (Especialmente necesario), $I$ 
(Importantemente necesario), $O$ (Ordinario en importancia), $S$ (sin importancia), y, $N$ (No deseable). Para esto, se tienen los pasos siguientes:

Primer paso, utilizando (1) se halla el número total de códigos de relaciones $(N)$ donde $n$ es el número de áreas del establecimiento, lo que permite establecer un límite en el número de veces en que puede utilizarse cada valor de cercanía, dado que el principiante y en ocasiones el experto, suele equivocarse indicando más codificaciones de un tipo, es decir, una persona puede indicar muchos valores " $A$ ".

$$
N=\frac{n(n-1)}{2}
$$

Segundo paso, aplicándose un análisis de Pareto se halla el porcentaje que no debe superarse en la asignación de un tipo específico de código, esto es como afirma Meyers y Stephens (2006): $A$ un $5 \%$ del total códigos, $E$ un $10 \%, I$ un $15 \%, y, O$ un $25 \%$.

- $\quad$ Razón de cercanía: Si bien es cierto, los motivos de cercanía no son un requisito indispensable para la construcción del diagrama de actividades, la realidad es que son de mucha utilidad, porque permiten que el diseñador, al igual que el usuario de la infraestructura no olvide los motivos por los cuales se decidió o no, colocar un área a cierta cercanía de otra. En la industria de alimentos, estas son dadas por las entidades gubernamentales, las cuales dictan los requisitos que las plantas deben seguir para operar en Colombia. Las razones de cercanía se tabulan siendo codificadas con números arábicos.

- $\quad$ Representación visual: Con base en los datos anteriores, se realiza el diagrama de relaciones de actividades para las áreas de los procesos que se han venido desarrollando.

\subsection{Fase 3: Diseño de la instalación de producción}

Esta es la fase final del estudio, en la cual se presenta un diseño en dos o tres dimensiones que atienda a los requerimientos señalados en el gráfico de relaciones de actividades y en el diagrama origendestino, siendo un esquema arquitectónico de la planta de procesos. Para esto, se aplica un software de diseño, en donde se hacen las notaciones de las áreas productivas.

\section{Resultados y Discusión}

\subsection{Aplicación de instrumentos y técnicas para la recolección de} información

\subsubsection{Diseño del cuestionario y medio de aplicación}

La estructura del cuestionario obedece a las consideraciones hechas por Estupiñan, (2005); Cámara de comercio de Bogotá, (2010); DANE, et al., (2013); y Cámara de comercio de Santa Marta (2017), quienes vienen diseñando excelentes guías para el levantamiento de información primaria de datos valiosos del sector agroindustrial. El cuestionario se hizo vía on-line por medio de las herramientas eficaces de Google, contando con dos secciones:

- Sección 1: Información de la empresa. Se pregunta por la razón social, tipos de productos que elabora, ubicación, nombre y cargo de quien realiza la encuesto. Esto entrega información del grado de capacitación profesional que tiene la persona encargada, y los productos que mayormente de producen en el sector.

- $\quad$ Sección 2: Fortaleza agroindustrial para el diseño de instalaciones. Aquí se pregunta por el volumen de producción obtenido mensualmente por producto, número de talento humano, tipo de tecnología utilizada, tecnología para el mejoramiento de la calidad, antigüedad de las máquinas de producción, y herramienta de la producción de la empresa. Esto determina la calidad del diseño, y la necesidad de propuestas para el sector. 


\subsection{Análisis de la información}

Se contactó vía telefónica a las 50 empresas de procesamiento de frutas y hortalizas ubicadas en la región Caribe, a través de la cual se les presentaron las solicitudes de información vía e-mail y la encuesta on-line que detallan la fidelidad del estudio. Sin embargo, sólo 11 entidades realizaron la encuesta en pro de contribuir a su sector (Tabla 1), esto evidencia cuan desligado esta el sector productivo de la academia y viceversa; así mismo, los establecimientos que respondieron se distribuyen así: El departamento del Atlántico contó con el 73\% de las empresas encuestadas; y los departamentos de Bolívar, Cesar y Córdoba con un 9\% cada uno. Lo cual pudo deberse gracias a la gran cantidad de empresas ubicadas en Atlántico, y a la accesibilidad de estas para brindar información de interés que beneficie a su sector.

En la Tabla 1, se puede apreciar un resumen singular de la encuesta, en donde los productos mayormente producidos en las plantas procesadora de frutas y hortalizas, son las pulpas y deshidra- tados. Además, el 63,7\% de las organizaciones son microempresas, lo cual se evidencia en la cantidad de toneladas producidas mensualmente por cada una de estas organizaciones que no superan las Seis (6) toneladas.

También se obtuvo que en cuanto a talento humano el 54,5\% de las empresas tienen hasta 10 trabajadores, $27,3 \%$ de once a cincuenta, y, el $18,2 \%$ de cincuenta y uno a doscientos empleados; referente al tipo de tecnología utilizada se halló que el 45,45\% emplea tecnología para pulpas, y, el $54,55 \%$ para deshidratado y molido; en tecnología para el mejoramiento de la calidad, se determinó que el 45,5 \% utiliza código de barras, el 45,5\% softwares, y el 9,1\% otros; en respuesta a la antigüedad de las máquinas de producción se encontró que en promedio las máquinas tienen 10,94 años; y en asociación a las herramientas de la producción de la empresa se obtuvo que el 54,5\% emplean pronósticos de ventas, el 27,3\% estimación de producción, el 9,1\% plan de requerimientos de materiales, y el 9,1\% ninguno.

\section{TABLA 1. EMPRESAS ENCUESTADAS Y PRODUCCIÓN MENSUAL EN TONELADAS (T)} FUENTE: ELABORACIÓN PROPIA

\begin{tabular}{|c|c|c|}
\hline Producto & Empresas & Producción en toneladas \\
\hline Pulpas de frutas & Tango juices S.A.S & 8 \\
\hline Pulpa de fruta congelada natural & Pulpa del Cesar & 2 \\
\hline Néctar de frutas & Conservas California & manzana-170 y pera-130 \\
\hline Pulpa de fruta congelada natural & Pulpas del caribe Montería & 3 \\
\hline $\begin{array}{l}\text { Pulpa de tamarindo, mora, corozo, } \\
\text { mango, cañandonga }\end{array}$ & El niño Roy tamarindo momposino & $\begin{array}{l}4 \text { tamarindo y } 1 \text { de mango, } \\
\text { mora, corozo y cañadonga. }\end{array}$ \\
\hline Crema de coco & Fábrica de confite dromedario S.A.S & 8 \\
\hline Vinagre; salsa negra; esencias & Productos alimenticios el tonel & 1,5 vinagres y salsa negra \\
\hline Caldos y condimentos & Condimar S.A. & 4 \\
\hline $\begin{array}{c}\text { Esencias, salsas y aderezos para } \\
\text { alimentos }\end{array}$ & Distribuciones y productos Lina S.A.S & 2 \\
\hline Salsas y sabores & Proquicol S.A.S & salsa ají 1 y salsa de ajo 1. \\
\hline Concentrado de coco y crema de COCO & Productos de la palma S.A.S & 0,15 deshidratado \\
\hline
\end{tabular}


Esto demuestra la necesidad que tiene el sector de contar con una herramienta aplicada, que sirva de orientación para llevar a cabo excelsos diseños de producción, permitiendo así, la tecnificación de sus procesos. Además, es apropiado realizar un diseño de instalaciones en el marco de las microempresas, porque la mayoría de organizaciones del sector manufacturero de hortalizas y frutas son de este tipo, por ello, a continuación, se prosigue con el diseño para una producción de $500 \mathrm{~kg} /$ hora como requerimiento principal.

\section{Determinación de los patrones de}

\section{flujo de materiales}

\subsection{Gráfico Origen-Destino o diagrama de-hacía entre máquinas}

Siguiendo con los métodos para realizar esta representación, en la Tabla 2 se encuentra la codificación de la maquinaria necesaria para obtener los productos de pulpa de mango, snacks de mango, rodajas de berenjena deshidrata, y ají en polvo. En la Tabla 3, se muestra las secuencias que siguen las materias primas a través de las máquinas para la obtención de los productos terminados (indicando así el flujo del proceso), junto con su frecuencia absoluta de viaje por la serie de máquinas correspondiente; dicha frecuencia fue establecida con base en la capacidad de producción que puede tener una microempresa de procesamiento de frutas y hortalizas, que cuenta con las capacidades nominales de estos equipos (Tabla 2).

Posteriormente se realiza el diagrama de-hacia (Tabla 4), en donde sus datos cuantitativos son la frecuencia de viaje que realizan los productos por las máquinas. La forma de obtener el contenido número de la Tabla 4, se explica a continuación con base en los autores Watana y Wiyarath (2011) y Lin, et al., (2013).

- $\quad$ Paso 1. Después de tener la ruta que siguen los productos junto a su frecuencia de viaje (Tabla 3). El aprendiz o el experto en diseño, señala los pares secuenciales de flujo que siguen los pro- ductos, por ejemplo: $\mathrm{AB}, \mathrm{BC}, \mathrm{CD}, \ldots$, nótese que no se escoge el par AC porque tal combinación no existe, lo mismo ocurriría con JH (Tabla 3).

- Paso 2. A los pares secuenciales señalados, se les asocian el número de veces en que los productos pasan por ellos, por ejemplo: La combinación de máquinas $\mathrm{AB}$ es tocada por la ruta de los productos 1, 2, 3 y 4, por lo que el número de veces en que pasan productos de la máquina A hacia la máquina B es 91 (Producto 1), 14 (Producto 2), 25 (Producto 3), y 24 (Producto 4), sumando estos valores se tienen 154 viajes totales en un mes. Lo mismo ocurre con las combinaciones $\mathrm{BC}$ y CD que son tocadas por los cuatro productos. Para los demás grupos se aplica la misma metodología.

- Paso 3. Las cantidades del paso anterior se insertan en la matriz del diagrama de-hacia, la diagonal de esta no posee datos numéricos porque no es lógico enviar productos en procesos que salen de una maquina a la misma máquina. La forma de colocar los datos dentro de la matriz es sencilla, se señala la fila correspondiente de la columna de entrada a la matriz que represente de donde se envía, luego se indica verticalmente la columna de la fila de entrada horizontal que simboliza hacia donde se envía el producto o material en proceso, la intercepción señala el lugar donde se coloca el valor numérico del par analizado. Para las demás interacciones se coloca el número cero $(0)$ pues no se hace viaje de-hacia.

\subsection{Diagrama de relaciones de actividades}

Valores de cercanía: La Tabla 5 presenta el número de áreas de trabajo de la empresa, en total 26, introduciendo este valor en (1) se tienen 325 códigos de relaciones. Después, con los porcentajes propios del análisis de Pareto indicados anteriormente en la metodología, se tiene la Tabla 6; se enfatiza que los códigos "N" y "S" se emplean en la asignación de las relaciones restantes. 
TABLA 2. CODIFICACIÓN DE LA MAQUINARIA

FUENTE: ELABORACIÓN PROPIA CON BASE A MECAFOOD S.A.S

\begin{tabular}{|c|c|}
\hline Código & Nombre de máquina \\
\hline A & Básculas (0-500 Kg) \\
\hline B & Seleccionador alimentador de rodillo (400 a $500 \mathrm{Kg} / \mathrm{hora}$ ) \\
\hline $\mathrm{C}$ & Lavadora de cepillos (500 Kg /hora) \\
\hline $\mathrm{D}$ & Escaldador de tornillos (500 kg/hora) \\
\hline $\mathrm{E}$ & Despulpadora horizontal (800 a 900 kg /hora) \\
\hline $\mathrm{F}$ & Clarificador $(500 \mathrm{~kg} / \mathrm{h})$ \\
\hline G & Desairador (500 kg/h) \\
\hline $\mathrm{H}$ & Pasteurizador unitubular (1.000 I/hora) \\
\hline 1 & Selladora y dosificadora semiautomática de viscosos (30 L) \\
\hline $\mathrm{J}$ & Lavadora por inmersión (400 a 500 kg /hora) \\
\hline $\mathrm{K}$ & Deshidratador a gas por convección (150 a 250 kg/baches) \\
\hline $\mathrm{L}$ & Selladora vertical industrial de pedal o neumática \\
\hline M & Molino de martillo (50 kg/h) \\
\hline
\end{tabular}

TABLA 3. RUTA DEL PRODUCTO A TRAVÉS DE LA MAQUINARIA JUNTO A SU FRECUENCIA DE FLUJO

FUENTE: ELABORACIÓN PROPIA

\begin{tabular}{|c|c|c|}
\hline Producto & Ruta por máquina & Número de veces/ mes \\
\hline 1.Pulpa de mango congelada & ABCDEFGHI & 14 \\
\hline 2.Snack de mango & ABCDJKL & 25 \\
\hline 3.Berenjena deshidratada & ABCDJKL & 24 \\
\hline 4.Ají molido & ABCDJKML & 14 \\
\hline
\end{tabular}

TABLA 4. DIAGRAMA DE-HACÍA PARA FLUJO DE PROCESO DE MÁQUINAS

\section{FUENTE: ELABORACIÓN PROPIA}

\begin{tabular}{c|c|c|c|c|c|c|c|c|c|c|c|c|c|}
\hline DE - HACIA & A & $\mathbf{B}$ & $\mathbf{C}$ & $\mathbf{D}$ & $\mathbf{E}$ & $\mathbf{F}$ & $\mathbf{G}$ & $\mathbf{H}$ & $\mathbf{I}$ & $\mathbf{J}$ & $\mathbf{K}$ & $\mathbf{M}$ & $\mathbf{L}$ \\
\hline $\mathbf{A}$ & - & 154 & 0 & 0 & 0 & 0 & 0 & 0 & 0 & 0 & 0 & 0 & 0 \\
\hline $\mathbf{B}$ & & - & 154 & 0 & 0 & 0 & 0 & 0 & 0 & 0 & 0 & 0 & 0 \\
\hline $\mathbf{C}$ & & & - & 154 & 0 & 0 & 0 & 0 & 0 & 0 & 0 & 0 & 0 \\
\hline $\mathbf{D}$ & & & & - & 91 & 0 & 0 & 0 & 0 & 63 & 0 & 0 & 0 \\
\hline $\mathbf{E}$ & & & & & - & 91 & 0 & 0 & 0 & 0 & 0 & 0 & 0 \\
\hline $\mathbf{F}$ & & & & & & - & 91 & 0 & 0 & 0 & 0 & 0 & 0 \\
\hline $\mathbf{G}$ & & & & & & & - & 91 & 0 & 0 & 0 & 0 & 0 \\
\hline $\mathbf{H}$ & & & & & & & & - & 91 & 0 & 0 & 0 & 0 \\
\hline $\mathbf{I}$ & & & & & & & & & - & 0 & 0 & 0 & 0 \\
\hline $\mathbf{J}$ & & & & & & & & & & - & 63 & 0 & 0 \\
\hline $\mathbf{K}$ & & & & & & & & & & & - & 24 & 39 \\
\hline $\mathbf{M}$ & & & & & & & & & & & & - & 24 \\
\hline L & & & & & & & & & & & & & - \\
\hline
\end{tabular}




\begin{tabular}{|c|c|}
\hline \multicolumn{2}{|c|}{ Áreas de trabajo } \\
\hline 1.Recepción y envío & 14. Inmersión \\
\hline 2.Pesaje Inicial & 15. Deshidratación \\
\hline $\begin{array}{l}\text { 3.Selección y } \\
\text { clasificación }\end{array}$ & 16. Reposo o enfriamiento \\
\hline 4. Control de calidad & 17. Adecuación y rebanado \\
\hline 5. Pesaje final & 18. Molienda \\
\hline 6. Pre-lavado y lavado & $\begin{array}{l}\text { 19. Bodega de deshidratados } \\
\text { terminados }\end{array}$ \\
\hline 7. Escaldado & 20. Cuarto de congelación \\
\hline 8. Despulpado & $\begin{array}{l}\text { 21. Bodega productos de } \\
\text { limpieza, papelería y demás }\end{array}$ \\
\hline 9. Clarificado & 22. Zona de residuos sólidos \\
\hline 10. Desairado & 23. Sanitarios y vestidores \\
\hline 11. Pasteurizado & 24. Cafetería \\
\hline $\begin{array}{l}\text { 12. Empaque, sellado } \\
\text { y embalaje }\end{array}$ & 25. Lavamanos \\
\hline 13. Moldeado & $\begin{array}{l}26 . \text { Bodega de insumos y } \\
\text { materiales }\end{array}$ \\
\hline
\end{tabular}

TABLA 6. LÍMITE NO PORCENTUAL DE CÓDIGO DE RELACIONES

FUENTE: ELABORACIÓN PROPIA

\begin{tabular}{|c|c|}
\hline Código & Límite \\
\hline $\mathrm{A}$ & 16 \\
\hline $\mathrm{E}$ & 33 \\
\hline $\mathrm{I}$ & 49 \\
\hline $\mathrm{O}$ & 81 \\
\hline
\end{tabular}

- Razón de cercanía: Dado que aquí se está realizando un diseño de distribución en planta para una infraestructura agroindustrial, existen ciertas resoluciones de entidades gubernamentales en Colombia que manifiestan requisitos de cercanía, a continuación, se exponen las notificaciones más relevantes:

- Los residuos sólidos "deben ser ubicados de manera tal que no representen riesgo de contaminación al alimento, a los ambientes o superficies de potencial contacto con este"
(Resolución 2674, 2013). Referente a los lavamanos, se exige que cuenten con grifos de accionamiento no manual dotados de los implementos de aseos necesarios, en las áreas de producción o próximas a ellas para mantener la higiene y la supervisión de las buenas prácticas (Resolución N²674, 2013).

- Los restaurantes y establecimientos gastronómicos, necesitan estar localizados en sitios secos, no inundables y en terrenos de fácil drenaje; No se podrán localizar junto a botaderos de basura, pantanos, ciénagas y sitios que puedan ser criaderos de insectos, roedores u otro tipo de plaga (Resolución $N^{\circ} 2674$, 2013). Con relación a los sanitarios, deben estar aislados de la zona de producción, con instalaciones adecuadas para hombres y mujeres por separado (Decreto 3075, 1997).

- El laboratorio debe estar ubicado de modo que no sea afectado por la contaminación cruzada, asegurando el control al acceso y el uso de las áreas que afectan a la calidad de los ensayos y/o de las calibraciones (NTC-ISO/ IEC $N^{\circ} 17025,2005$ ).

Estos requisitos se materializaron en los códigos de cercanía que se encuentran en la Tabla 7, para la construcción del diagrama de afinidad de actividades (relaciones de actividades).

\section{TABLA 7. MOTIVOS DE CERCANÍA FUENTE: ELABORACIÓN PROPIA}

\begin{tabular}{c|c} 
Código & Razón \\
\hline 1 & Flujo de materia en proceso \\
\hline 2 & Flujo de personal \\
\hline 3 & NTC-ISO-IEC 17025 \\
\hline 4 & Contaminación \\
\hline 5 & Humedad \\
\hline 6 & Higiene \\
\hline 7 & Facilidad de contacto y supervisión \\
\hline
\end{tabular}




\section{Figura 1. Diagrama de relación de actividades}

Fuente: Elaboración propia

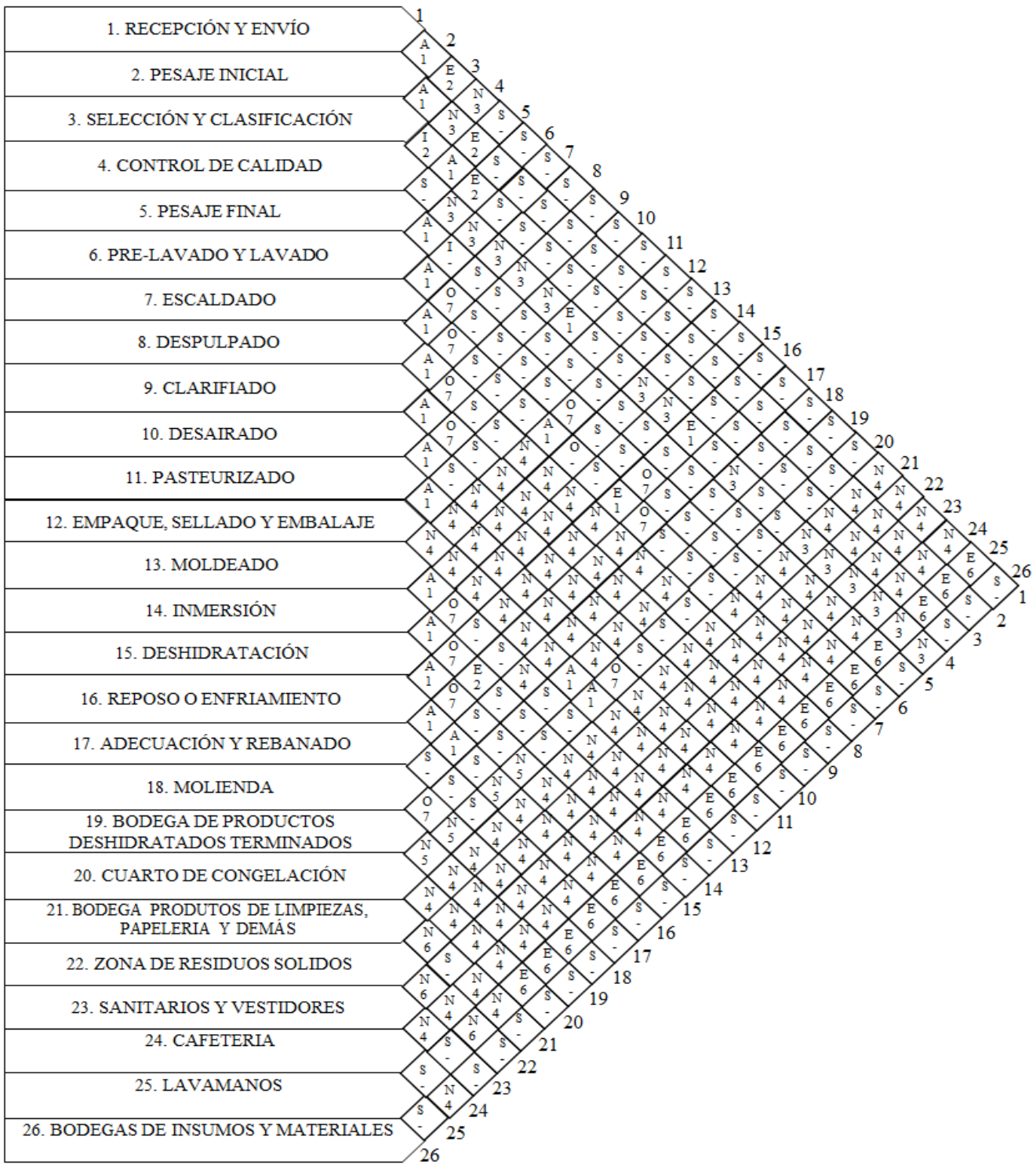

- $\quad$ Representación visual: Con ayuda de los datos anteriores, se realiza el diagrama de relaciones de actividades como se muestra en la Figura $\mathbf{1}$, en este se visualiza la gran cantidad de relaciones que debe cumplir un arreglo mínimo para un buen diseño de instalaciones, lo que se debe gracias al número de normativas gubernamentales que rigen el sector. Así mismo, se puede apreciar comparativamente que este diagrama de actividades coincide con la Matriz De-Hacia (Tabla 4).

\subsubsection{Diseño de la instalación de producción}

Los datos anteriores tienen la finalidad de proporcionar la información necesaria para realizar un buen diseño de producción, dado que la matriz de origen destino y el diagrama de actividades, son 
insumos valiosos para atender los requerimientos funcionales y no funcionales. Por esto, en la Figura 2 se tiene el diseño de la planta agroindustrial hortofrutícola en respuesta a las necesidades del sector.

En este diseño de instalaciones (Figura 2) se observa que existe un acceso central que comienza con la "zona de cargue y descargue" y termina con el "escaldado", estas son las actividades mixta de los procesos de despulpado y deshidratado; también se aprecia que hay tres líneas punteadas que toman sentidos diferentes, la azul, representa la secuencia que sigue el mango por las diferentes actividades hasta su conservación como pulpa de mango en el cuarto congelado a una temperatura de $-4^{\circ} \mathrm{C}-2^{\circ} \mathrm{C}$; la roja, muestra el recorrido que realiza la berenjena y el ají, para ser guardados en la bodega como rodajas de berenjena deshidratada y ají en polvo (productos terminados); y la línea verde, que llega hasta pesaje final y se dirige a bodega, hace alusión a la materia prima que ingresa a la empresa, pero al no necesitarse en el momento se guarda hasta la próxima producción.

\section{Figura 2. Diseño de instalación para la planta agroindustrial hortofrutícola} Fuente: Elaboración propia

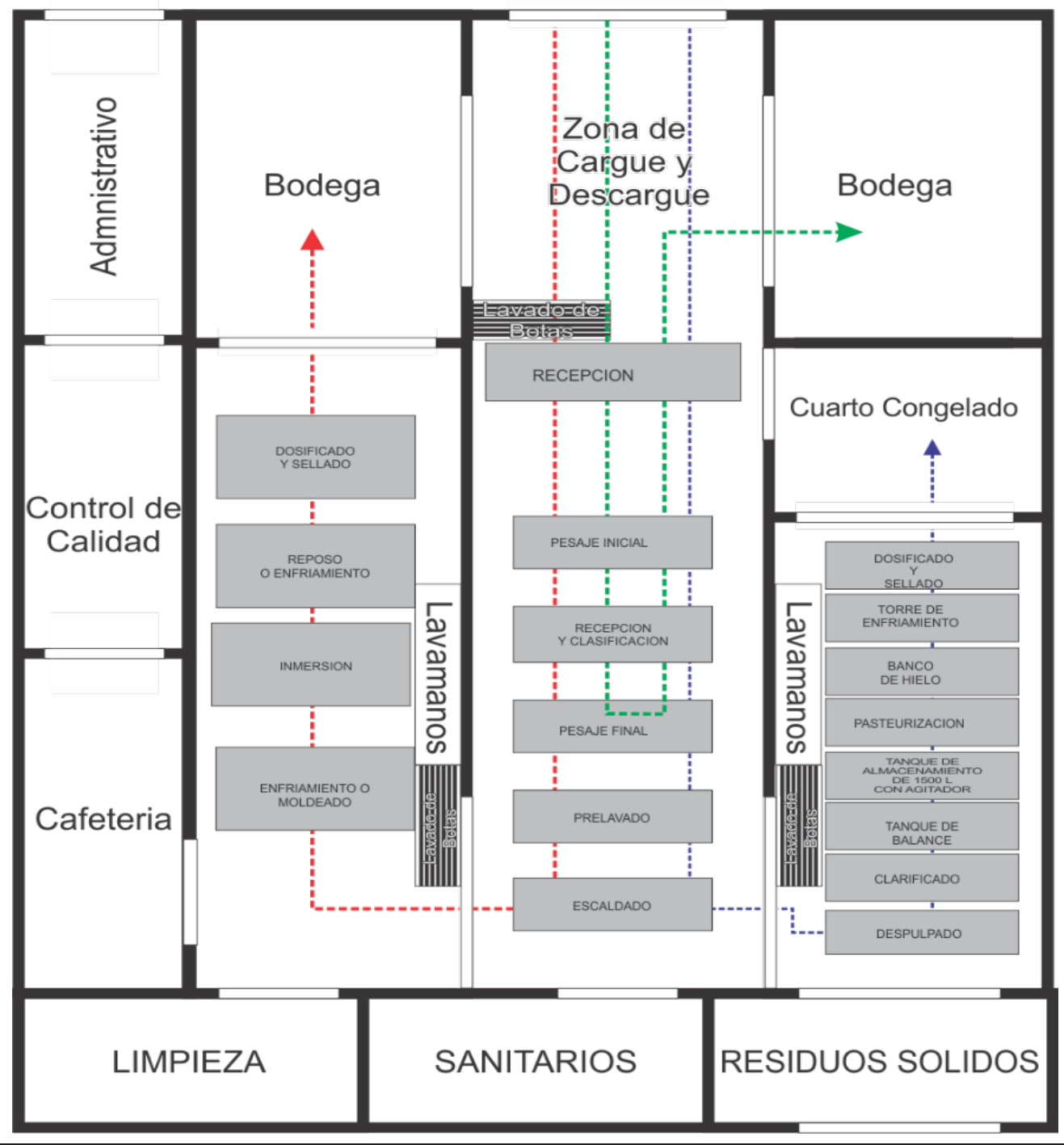


La Figura 2 también contempla los lavamanos en las líneas de proceso de despulpado y deshidratado, para evitar que el personal se mueva demasiado y genere contaminación cruzada. Los demás arreglos son propios de los requisitos de las entidades de salubridad y de la lógica del proceso, los sanitarios, la disposición de recursos sólidos y la bodega de limpieza están alejados de la zona productiva. Finalmente, se tienen las áreas administrativas y la zona de cafetería aisladas del proceso, por motivos de higiene y seguridad microbiana, y se define el espacio del control de calidad con conexión al área productiva por la necesidad de las tomas de muestras para valorar la calidad de la materia prima (mango, ají o berenjena), y la de los productos terminados.

\section{Conclusiones}

El diseño de instalaciones es una herramienta poderosa para el sector productivo, al proporcionar un esquema arquitectónico que ayuda a muchas organizaciones a estar acorde con las exigencias estatales y/o gubernamentales que rigen su industria; no obstante, la aplicación de este, debe realizarse a medida que se conozcan los requerimientos y necesidades del sector u objeto de estudio.

Es indispensable que el diseñador (o grupo de diseñadores) establezca el instrumento de captura que permita justificar la importancia de realizar el estudio; lo cual, puede hacerse por medio de un cuestionario que este enfocado a detectar falencias del sector o empresa en materia de diseño y productividad.

Posteriormente, se deberá efectuar estudios que ofrezcan un esquema de la secuencia del proceso para alcanzar el producto terminando, la necesidad de maquinarias y equipos, así como la frecuencia de utilización de las tecnologías.

A su vez, es recomendable realizar un análisis de las relaciones que tendrán las diferentes máquinas y áreas de la empresa, especificando los motivos de estas asociaciones, para dar una buena argumentación al arreglo propuesto de la planta. Además, es indispensable realizar los diagramas y gráficos de estas interacciones junto a sus razones para obtener una ayuda ingenieril aplicable, efectuándose con ello un diseño de la instalación sobre un plano en dos o tres dimensiones.

Por último, se resalta que este artículo es un buen soporte metodológico y teórico para futuros estudios y aplicaciones dentro del sector productivo, como el agroindustrial hortofrutícola que carece de este tipo de estudios; contribuyendo de esta forma a futuras investigaciones en el diseño de instalaciones para el mejoramiento de la producción de este sector, entre otros.

\section{Referencias}

Asociación Hortofrutícola de Colombia. (2016). Balance del sector hortofrutícola en 2016. Disponible en: http://www.asohofrucol.com.co/ [Consultado 3011-2018]

Cámara de comercio de Bogotá. (2010). Caracterización del sector de industrias culturales y creativas en Bogotá y Cundinamarca. Bogotá D.C., Colombia: Cámara de comercio de Bogotá.

Cámara de comercio de Santa Marta. (2017). Coyuntura empresarial del Magdalena enero a junio del 2017. Santa Marta D.T.C.H., Colombia: Cámara de comercio de Santa Marta.

Campo-Arias, A., Sanabria, A. R., Ospino, A., Guerra, V. M., Caamaño, B. H. (2016). Polivictimización por el conflicto armado y sufrimiento emocional en el Departamento del Magdalena, Colombia. Revista Colombiana de Psiquiatría, 46 (32), julio-septiembre, pp.147-153.

Causado, E; Reatiga, I. (2013). Cadena logística de subproductos residuales en la industria de tajada de plátano para exportación" Revista Dimensión Empresarial, (11)2, Julio-Diciembre, pp. 9-16.

Causado-Rodríguez, E., Díaz, A. F.; Sánchez, G. D. (2018). Reubicación de Instalaciones productivas mediante método matemático de recálculo de coordenadas MMRC. Revista Clío América, 12(23), Enero-Junio, pp. 61-72.

Clavijo, S., Vera, A., Fandiño, A. (2012). La desindustrialización en Colombia Análisis cuantitativo de sus determinantes. Bogotá D.C., Colombia: ANIF. 
Corena, L., Cujia, D. (2012). Diseño de una propuesta de mejoramiento del sistema productivo de la empresa BANANITAS SAS de Santa Marta D.T.C.H, tesis (pregrado en ingeniería industrial), Santa Marta D.T.C.H. - Colombia, Universidad del Magdalena.

DANE-Departamento Nacional de Estadístico ; MADR-Ministerio de Agricultura ; CIAT - Desarrollo Rural ; Centro Internacional de Agricultura Tropical. (2013). Censo de plantas procesadores de yuca para uso industrial resultados. Disponible en https:// www.dane.gov.co/ [Consulta 17-11-18].

Decreto $N^{\circ} 3075$. Ministerio de Salud, Bogotá, Colombia, 23 de diciembre de 1997.

Departamento Administrativo Nacional de Estadística. (2017a). Encuesta Nacional Agropecuaria ENA -2016. Disponible en https://www.dane.gov.co/ [Consultado 15-11-2018]

Departamento Nacional de Planeación. (2015). Conpes 3850 Fondo Colombia en Paz. Consejo Nacional de Política Económica y Social. Disponible en: https:// colaboracion.dnp.gov.co/CDT/Conpes/Económicos/3850.pdf [Consultado 30-11-2018]

Departamento Nacional de Planeación. (2004). Cadenas productivas, estructura, comercio internacional y protección. Bogotá D.C. Colombia: Departamento Nacional de Planeación, pp. 107-108.

Estupiñan, A. (2005). Diseño de indicadores de producción en la industria de alimentos de Barranquilla y Cartagena, 3er congreso de Avances en Ingeniería y tecnología, Cartagena de Indias, Colombia: LACCET 2005.

Gobernación del Magdalena. (2016). Plan de Desarrollo 2016 - 2019 Departamento del Magdalena - Magdalena Social, ¡Es la Vía!. Santa Marta D.T.C.H., (Magdalena): Gobernación del Magdalena, http://www. magdalena.gov.co/. [Consulta, 13-12-2018].

Heragu, S. (2016). Facilities design. $3^{\text {ra }}$ Edición. United States of America: CRC Press.

Instituto Nacional de Vigilancia de Medicamentos y Alimentos. (2017). Censo de plantas productoras de alimentos regional. Disponible en https://www. icbf.gov.co/ [Consulta 20-11-2018].

INVEMAR-Instituto de Investigaciones Marinas y Costeras José Benito Vives de Andréis. (2004). Informe del Estado de los Ambientes Marinos y Costeros en Colombia. Disponible en https://http://www.invemar.org.co/ [Consultado 19-10-2018].
Kusiak, A., Heragu, S. S. (1987). The facility layout problem. European Journal of operational research, 29(3), Junio, 229-251.

Lin, Q. L., Liu, H. C., Wang, D. J., Liu, L. (2013). Integrating systematic layout planning with fuzzy constraint theory to design and optimize the facility layout for operating theatre in hospitals. Journal of Intelligent Manufacturing, 26(1), pp. 87-95.

MADR-Ministerio de Agricultura y Desarrollo Rural; FNFH- Fondo Nacional de Fomento Hortofrutícola; ASOHOFRUCOL - Asociación Hortofrutícola de Colombia; SAG- Sociedad de Agricultores y Ganaderos del Valle de Cauca. (2006). Plan Frutícola Nacional Diagnóstico y análisis de los recursos para la fruticultura en Colombia. Cali, Colombia: Impresora Feriva, pp. 47-49.

MADR-Ministerio de Agricultura y Desarrollo Rural. (2016b). Cadena de mango indicadores e instrumentos. Disponible en https://www.minagricultura.gov.co/ [Consultado 15-11-2018]

Maina, E., Muchiri, P., Keraita, J. (2018). Improvement of Facility Layout Using Systematic Layout Planning. IOSR Journal of Engineering (IOSRJEN), 8 (5), pp. 33-43.

Martínez, C., Soto, M., Magaña, J., Lincón., L; Kiessling, C. (2015). Estudio técnico del proyecto TOMATO-MIX. Revista Mexicana de Agronegocios, 19 (36), EneroJunio, pp.1275-1285.

Meisel-Roca, A., Pérez-Valbuena, G. J. (2006). Geografía física y poblamiento en la Costa Caribe colombiana. Cartagena de Indias, Colombia: Banco de la República de Colombia Documentos de Trabajo.

Meyers, F., Stephens, M. (2006). Diseño de instalaciones de manufactura y manejo de materiales. (3ra.ed). México: Pearson Educación.

Nariño, A. H., León, A. M., Rivera, D. N. (2010). Criterios para la elaboración de mapas de procesos. Particularidades para los servicios hospitalarios. Revista Ingeniería Industrial, 30(2), Julio, pp. 1-7.

Niebel, B. W., Freivalds, A., Osuna, M. A. G. (2004). Métodos, estándares y diseño del trabajo. México D.F., México: Alfaomega.

NTC-ISO/IEC N ${ }^{\circ}$ 17025. Requisitos Generales para la Competencia de los Laboratorios de Ensayos y Calibración, Bogotá, Colombia, 26 de octubre de 2005.

Ojaghi, Y., Khademi, A., Yusof, N. M., Renani, N. G., Hassan, S. A. H. B. S. (2015). Production layout optimization 
for small and medium scale food industry. In Procedia CIRP, 26, pp. 247-251.

Palacio, A.E. (2014). Agroindustria, una apuesta por la innovación y el progreso. Revista Frutas \& Hortalizas, (35), julio-septiembre, pp.5.

PROCOLOMBIA. (2016). El mundo invierte en Colombia Inversión en el sector agroindustrial. Bogotá D.C.Colombia: Procolombia.co.

Resolución $N^{\circ}$ 1471. Ministerio de Salud República de Colombia, Bogotá D.C., Colombia, 12 de octubre de 1984.

Resolución № 2674. Ministerio de Salud y protección social, Bogotá, Colombia, 22 de julio de 2013.

Stephens, M., Meyers, F. (2013). Manufacturing facilities design and material handling. West Lafayette, United State: Purdue University Press, pp. 130-200.

Sugiyono, A. (2006). Cellular manufacturing system aplication on redesign production layout with using heuristics algorithm. $2^{\circ}$ IEEE International Conference on Management of Innovation and Technology, 2006 IEEE International Conference, 21-23 Junio 2006, Singapore, China, pp. 940-944.

Tak, C. S., Yadav, L. (2012). Improvement in Layout Design using SLP of a small size manufacturing unit: A case study. IOSR Journal of Engineering, 2(10), Octubre, pp.1-7.

Watanapa, A., Wiyaratn, W. (2011). Systematic Layout Planning to Assist Plant Layout: Case Study Pulley Factory. Applied Mechanics and Materials, 110-116, Octubre, pp.3952-3956.

Causado Rodríguez, E.; Ospino Ayala, 0.J.; Racedo Gutiérrez, J.P. (2019). Diseño de producción para la agroindustria hortofrutícola de pulpas congeladas, deshidratados, y/o moliendas en la región Caribe. Revista EIA, 16(32), JulioDiciembre, pp. 113-127. [Online]. Disponible en: https://doi. org/10.24050/reia.v16i32.1284 